\title{
Incidencia de la justicia sobre el capital psicológico y la satisfacción laboral en trabajadores de la industria automotriz
}

\section{Nayeli Alejandra Avitia-Cordero, Paulina Irasema Salas-Guevara, María Marisela Vargas-Salgado, Karla Gabriela Gómez-Bull}

\author{
Generalista de Recursos Humanos \\ Generalista de Recursos Humanos \\ Departamento de Ciencias Administrativas \\ Universidad Autónoma de Ciudad Juárez \\ Departamento de Ingeniería Industrial y Manufactura \\ Universidad Autónoma de Ciudad Juárez
}

En la actualidad, las organizaciones tienen la necesidad de contar con personas comprometidas, calificadas y satisfechas. Por ello, cada día las empresas se esfuerzan en ofrecer entornos justos y competitivos con la finalidad de ser exitosas. El objetivo de esta investigación fue analizar si la justicia organizacional impacta sobre el capital psicológico y la satisfacción laboral de los empleados de nivel operativo que laboran en una empresa del ramo automotriz en la frontera norte de Chihuahua. El trabajo fue de tipo cuantitativo, no experimental, transversal y correlacional. Se consideró una muestra probabilística y se aplicaron 329 encuestas a trabajadores de nivel operativo, las cuales fueron suministradas en el entorno de trabajo. Los datos recabados fueron analizados mediante la estimación de mínimos cuadrados parciales (PLS). El modelo se corrió con el software Smart PLS3. Los resultados evidencian que las relaciones planteadas son significativas: la justicia incide positiva y significativamente sobre la satisfacción laboral y el capital psicológico. Además, el capital psicológico influye significativamente sobre la satisfacción laboral.

Palabras clave: justicia organizacional, capital psicológico, satisfacción laboral, industria manufacturera

\section{Impact of justice on psychological capital and job satisfaction in workers in the automotive industry}

Nowadays, organizations need to have committed, qualified and satisfied people, that is why day by day companies strive to offer fair and competitive environments in order to be successful. The objective of this investigation was to analyze whether organizational justice impacts the psychological capital and job satisfaction of employees at the operational level, who work in a company in the automotive industry on the northern border of Chihuahua. The work was of a quantitative, non-experimental, cross-sectional and correlational type. A probabilistic sample was considered and 329 surveys were applied to workers at the operational level, which were provided in the work environment. The data collected was analyzed by estimating partial least squares [PLS]. The model was run with Smart PLS3 software. The results show that the relationships proposed are significant: justice positively and significantly affects job satisfaction and psychological capital, at the same time that psychological capital significantly influences job satisfaction.

Keywords: organizational justice, psychological capital, job satisfaction, manufacturing industry 


\section{Impacto da justiça no capital psicológico e na satisfação do trabalho em trabalhadores da indústria automotiva}

Atualmente, as organizaçôes precisam ter pessoas comprometidas, qualificadas e satisfeitas, e é por isso que as empresas diariamente se esforçam para oferecer ambientes justos e competitivos para serem bem-sucedidos. $\mathrm{O}$ objetivo desta pesquisa é analisar se a justiça organizacional afeta o capital psicológico e a satisfação no trabalho dos funcionários no nível operacional, que trabalham em uma empresa do setor automotivo na fronteira norte de Chihuahua. $\mathrm{O}$ trabalho foi do tipo quantitativo, não experimental, transversal e correlacional. Uma amostra probabilística foi considerada e 329 pesquisas foram aplicadas aos trabalhadores no nível operacional, que foram feitas no ambiente de trabalho. Os dados coletados foram analisados estimando-se os mínimos quadrados parciais [PLS]. O modelo foi executado com o software Smart PLS3. Os resultados mostram que as relaçôes propostas são significativas: a justiça afeta positiva e significativamente a satisfação no trabalho e o capital psicológico, ao mesmo tempo em que o capital psicológico influencia significativamente a satisfação no trabalho.

Palavras-chave: justiça organizacional, capital psicológico, satisfação no trabalho, indústria de transformação

\section{Introducción}

Desde hace un tiempo, en el contexto empresarial, ha quedado claro que uno de los recursos más importantes es el humano, ya que contribuye considerablemente en la calidad, productividad y competitividad de la organización. Por ello, se considera necesario contar con empleados satisfechos (Alvarez, de Miguel, Noda, Alvarez, \& Galcerán, 2016). Sin embargo, últimamente, tanto las organizaciones como los empleados están expuestos a nuevos desafíos, por lo que se requiere que ambos se ajusten a los cambios con la finalidad de lograr los objetivos organizacionales (Jiménez, Jara, \& Celis, 2012). Cabe resaltar que la psicología organizacional ha tomado relevancia en los últimos años en el contexto sociolaboral (Dipp, Flores, \& Gutiérrez, 2010).

Hoy en día, en el contexto organizacional, es más frecuente que los empleados perciban la forma en que son valorados tanto por la empresa como por el superior, es decir, si son tratados de una forma justa en el trabajo (López, del Río, \& Villagrasa, 2018). Se tratan de eventos que el individuo experimenta durante el ciclo de vida laboral (Bouazzaoui, Wu, Roehrich, Squire, \& Roath, 2020). Por otra parte, el sistema de un contexto de justicia fortalece el capital psicológico del empleado, dado que el individuo utiliza las habilidades y capacidades para cumplir con éxito las actividades al estar seguro de que las posee (Bogler $\&$ Somech, 2019).

Cabe señalar que es imprescindible que los directivos se preocupen por los empleados; además, deben buscar el bienestar de estos con el propósito de que los sujetos se sientan contentos y satisfechos en su lugar de trabajo, ya que, a través de estas acciones, las personas tienden a encontrarse más adaptables y disponibles, lo que tiene un impacto en el éxito empresarial (Peña, Olloqui, \& Aguilar, 2013). Hablar de satisfacción laboral es de gran importancia, ya que señala la capacidad que tienen las organizaciones para saciar las necesidades de los empleados (Martín, 2006). Además, la satisfacción laboral tiene un efecto considerable en el desempeño, el bienestar y la salud de los empleados (Pérez, Herrera, Torres, \& Hernández, 2014). 
En México, la Industria Manufacturera, Maquiladora y de Servicios de Exportación (en adelante, IMMEX) es una de las industrias más importantes que ha cobrado gran relevancia en los últimos tiempos. Este tipo de industria es una fuente para el desarrollo económico del país (Bautista \& Peralta, 2017). En la frontera norte de Chihuahua, se encuentra la segunda ciudad más importante en este tipo de industria: según Index Juárez (2020), en esa zona se encuentra el mayor número de empresas que operan bajo el esquema IMMEX, con un 65\% del total a nivel estatal. Cabe destacar que el 32\% del total de empresas corresponde específicamente a la industria automotriz. Concretamente, en este tipo de industria, la percepción de justicia, el comportamiento de los empleados y la satisfacción en el trabajo son muy importantes, ya que estos aspectos se verán reflejados directamente en los resultados operacionales y el éxito de la organización. Por ello, de acuerdo con lo anterior, el objetivo de esta investigación es analizar si la justicia organizacional impacta sobre el capital psicológico y la satisfacción laboral de los empleados de nivel operativo que laboran en una empresa del ramo automotriz.

\section{Marco teórico}

\subsection{Justicia organizacional}

Desde hace un tiempo, las organizaciones consideran que la justicia debe prevalecer en el contexto organizacional, ya que la percepción que los empleados tienen con respecto a este constructo puede ser relevante para el logro de los objetivos organizacionales de la empresa para la que laboran (Mladinic \& Isla, 2011). Cada vez más, las personas se preocupan por encontrar justicia en diferentes actividades, contextos y etapas de la vida (Montalbán, Lugo, \& Soria, 2014). Concretamente, en el entorno laboral, la justicia organizacional es con- siderada como la equidad que se aplica en el lugar de trabajo, es decir, se espera que la organización empleadora trate a los trabajadores de manera justa y equitativa (Greenberg, 1990).

De acuerdo con algunos autores, el constructo "justicia organizacional” está compuesto por las dimensiones de justicia distributiva, procedimental, interpersonal e informacional. La justicia distributiva se refiere a la incertidumbre que las personas tienen al trato igualitario con respecto a las remuneraciones que reciben (Cropanzano, Bowen, \& Gilliland, 2007; Mladinic \& Isla, 2002); la procedimental, a la percepción de los empleados sobre cómo los procedimientos se aplican de manera apropiada e igualitaria en beneficio de ellos (Mladinic \& Isla, 2002; Pérez et al., 2014); la interpersonal, al trato justo y digno con el que los superiores se relacionan y dirigen hacia los trabajadores (Colquitt, 2001; Mladinic \& Isla, 2002); y la informacional, a la percepción que los empleados tienen con respecto a cómo se les proporciona información sobre las decisiones que se toman (Colquitt, 2001; Mladinic \& Isla, 2002).

En el contexto organizacional, cuando los empleados perciben que reciben un trato digno, prestaciones $y$ salarios justos, promociones y tareas equitativas, habrá un efecto en el desempeño de los trabajadores: mantendrán actitudes positivas y, por ende, obtendrán buenos resultados (De Boer, Bakker, Syroit, \& Schaufeli, 2002; Mladinic \& Isla, 2011). La justicia organizacional puede generar grandes beneficios para las firmas, ya que, si estas forjan una mayor confianza y compromiso en los trabajadores, estos mismos contribuirán en gran medida en los resultados de la empresa. Además, puede incidir en la reducción de los desacuerdos laborales que se pueden presentar en el entorno de trabajo (Cropanzano et al., 2007). En contraste, cuando los empleados perciben injusticia, los resulta- 
dos se verán afectados considerablemente. Incluso, los trabajadores pueden abandonar la organización por lo que perciben sobre el entorno laboral. Los empleados pueden expresar emociones y comportamientos negativos, además de sentimientos de insatisfacción y desmotivación (De Boer et al., 2002; Mladinic \& Isla, 2002, 2011). Cabe destacar que la percepción que los trabajadores tienen con respecto a las buenas prácticas de equidad internas puede ser benévola para la organización al tener un impacto considerable en los resultados de la operación (Pérez et al., 2014).

Cuando los empleados perciben un entorno de justicia por parte de los superiores, esto se manifestará en el comportamiento positivo de las personas y los resultados serán significativos (Karam et al., 2019). Además, la justicia se verá reflejada en la satisfacción de los trabajadores, ya que estos se esforzarán por lograr un buen desempeño y por permanecer en la organización (Wright \& Bonett, 2007). Al mismo tiempo, los empleados presentarán una mejor salud mental, ya que les agradará el contexto laboral en el que se desenvuelven (Martín, 2006; Shibaoka et al., 2010). Por el contrario, cuando prevalece la injusticia, la vida de los empleados se verá perjudicada y los trabajadores estarán insatisfechos con lo que realizan; es decir, realizarán acciones negativas en contra de los compañeros o incluso con el superior, y se opondrán a realizar cualquier cambio (Heidari \& Saeedi, 2012). Entonces, la justicia organizacional contribuye a crear satisfacción en los empleados, pues, cuando estos perciben que tanto los procesos como los resultados son justos, los sentimientos serán positivos y la satisfacción será mayor (Lambert, Keena, Leone, May, \& Haynes, 2020). De acuerdo con lo anterior, en esta investigación, se postula lo siguiente:

H1: La justicia incide positiva y significativamente sobre la satisfacción laboral.
Por otra parte, si los empleados son tratados con respeto y equidad por parte de los superiores, el capital psicológico aumenta y se fortalece cuando las personas son recompensadas y respetadas por lo que ellos realizan como parte de un resultado; es decir el capital psicológico no aumentará si solo se participa en el proceso y no hay un resultado (Eom, Hwang, \& Song, 2016). Además, la percepción de justicia en el lugar de trabajo induce a los empleados a tener comportamientos positivos que serán benévolos para la organización, ya que los empleados expondrán una mayor satisfacción (Singh \& Singh, 2019). Por lo anterior, se postula lo siguiente:

H2: La justicia incide positiva y significativamente sobre el capital psicológico.

\subsection{Capital psicológico}

Últimamente, las empresas consideran el capital psicológico como un constructo relevante, ya que contempla el comportamiento de los trabajadores (Azanza, Domínguez, Moriano, \& Molero, 2014). Se cree que es un factor importante que contribuye directamente en los resultados relacionados con el trabajo (Paek, Schuckert, Kim, \& Lee, 2015), ya que el comportamiento que el empleado manifiesta es fundamental para su buen desempeño en la organización (Luthans, Youssef, \& Avolio, 2007). En un entorno competitivo, se considera significativo el capital financiero, humano y social. Frente a ello, el capital psicológico implica ir más allá de lo tradicional, ya que se asocia con comportamientos y actitudes de las personas que se consideran vitales para el éxito de la empresa (Luthans, Luthans, \& Luthans, 2004). Además, la utilización adecuada del personal puede ser esencial en la ventaja competitiva organizacional (Corcione, 2015).

El "capital psicológico" se define como la situación psicológica que prevalece para realizar una actividad

Contabilidad y Negocios (16) 31, 2021 / e-ISSN 2221-724X 
o desafío con seguridad, y con una actitud optimista ante la posibilidad de que se puedan presentar triunfos en ese momento o más adelante sin rendirse ante los objetivos planteados; es decir, implica tener confianza (autoeficacia), hacer atribuciones positivas (optimismo), perseverar hacia metas (esperanza) y alcanzar el éxito ante adversidades (resiliencia) (Luthans, Avolio, Avey, \& Norman, 2007; Luthans et al., 2007). Algunos autores consideran que el capital psicológico constituye un estado que se caracteriza por la seguridad para realizar un trabajo complejo, y asumir las responsabilidades y consecuencias de la tarea encomendada (Omar, Salessi, \& Urteaga, 2014; Salessi \& Omar, 2017).

El capital psicológico es considerado como una fuente de emociones y comportamientos positivos que impacta directamente en la satisfacción de los empleados (Saraswati, 2019). Cuando los trabajadores están satisfechos con su trabajo, presentan comportamientos que benefician a la organización (Singh $\&$ Singh, 2019). Se cree que el capital psicológico se encuentra asociado a grandes niveles de satisfacción laboral (Perugini \& Solano, 2019). Por el contrario, cuando no está presente, los empleados experimentan insatisfacción en el lugar de trabajo (Saraswati, 2019). De acuerdo con lo anterior, se postula lo siguiente:

H3: El capital psicológico influye significativamente sobre la satisfacción laboral.

\subsection{Satisfacción laboral}

En el contexto organizacional, la satisfacción laboral es un constructo de gran relevancia, dado que, a través de ella, se logran resultados favorables: la actitud de los empleados se basa en creencias y valores personales, que se fortalecen en el área laboral; es decir, mientras mayor sea la satisfacción, mayor será el compromiso que el empleado tendrá con el trabajo (Singh \& Lon- car, 2010). Cabe destacar que la buena imagen que posee una empresa es un referente del buen clima y entorno que los empleados perciben con respecto al contexto laboral (Salazar, Cosiun, \& Torres, 2019).

El constructo de satisfacción laboral es definido como el conjunto de actitudes que la persona tiene hacia el trabajo (Robbins, 2004) y la postura que tiene el empleado con respecto a las diversas situaciones laborales (Bravo, Peiró, \& Rodríguez, 1996). Además, algunos autores afirman que es la impresión que el individuo tiene respecto de la ocupación y satisfacción que siente en relación con el empleo en el lugar de trabajo (Newstrom, 2011; Robbins \& Judge, 2009).

Una de las teorías más relevantes con respecto a la satisfacción es la de Herzberg, que está relacionada con los factores del trabajo tanto higiénicos como de motivación (Ewen, Smith, \& Hulin,1966). En el primer grupo de factores, se encuentran la comunicación, la jornada laboral, los riesgos de trabajo y las condiciones físico-ambientales. En el segundo grupo de factores, se encuentran el reconocimiento, la responsabilidad y la libertad de decidir cómo realizar el trabajo, entre otros (Ewen, Smith, \& Hulin, 1966). Entonces, los factores de motivación o intrínsecos son facetas muy específicas que propician la satisfacción del empleado en el entorno laboral (Gabriunas, 2010). No obstante, se pueden presentar situaciones que pueden afectar la satisfacción del empleado, como condiciones inadecuadas, incidentes o accidentes laborales, trato injusto, inestabilidad laboral, mala salud, falta de comunicación, carga excesiva de trabajo, o pago y beneficios inadecuados; además, tales eventos tienen un impacto en el desempeño y productividad de los sujetos (Escajadillo, 2013; Salazar et al., 2019). Así, los individuos insatisfechos se ausentan del trabajo frecuentemente y, por lo general, tienden a abandonar la organización (Martín, 2006) 


\section{Metodología}

La metodología de este trabajo es de tipo cuantitativa, no experimental, transversal y correlacional. La muestra fue probabilística y se consideraron como sujetos de estudio a operadores de producción. La investigación se llevó a cabo en una empresa de la industria maquiladora de la frontera norte de Chihuahua, específicamente, en el sector automotriz. En total, se aplicaron 329 encuestas. El instrumento fue administrado de forma directa a cada uno de los participantes en el contexto laboral.

Cada uno de los constructos se valoró a partir de la adaptación de instrumentos validados e identificados en la literatura. Se utilizó una escala tipo Likert de cinco opciones de respuesta. La justicia organizacional se valoró con dieciséis ítems a partir de la propuesta de Colquitt (2001); el capital psicológico se midió con veintiún preguntas a partir de la adaptación del instrumento de Luthans et al. (2007); y la satisfacción laboral fue evaluada por medio de veintidós ítems a partir de la adaptación de la propuesta de Meliá y Peiró (1989). Una vez recabada la información, los datos fueron sistematizados en una hoja de cálculo de Excel. Posteriormente, se analizaron los datos descriptivos a través de la herramienta estadística SPSS, mientras que los inferenciales se examinaron mediante la estimación de mínimos cuadrados parciales (PLS). El modelo se examinó con el software de análisis estadístico Smart PLS3.

\section{Resultados}

Las características de los participantes se exhiben en la tabla 1 , en la que se puede apreciar que el 55,9\% de los sujetos corresponde a mujeres y el $44,1 \%$ a hombres. Además, el 56,6\% dice tener entre 18 a 33 años. Con respecto a la antigüedad en la organización, casi el $60 \%$ tiene menos de 3 ańos laborando en ella. Por otro lado, 54,4\% cuenta con estudios de secundaria. Cabe destacar que el $100 \%$ de los sujetos de estudio se desenvuelven como operadores de producción en la industria automotriz.

Tabla 1. Características de los participantes $(\mathrm{n}=329)$

\begin{tabular}{|l|c|c|l|c|c|}
\hline \multicolumn{1}{|c|}{ Característica } & Personas & $\%$ & \multicolumn{1}{c|}{ Característica } & Personas & $\%$ \\
\hline Género & & & Edad & & \\
\hline Hombre & 145 & 44,1 & Entre 18 a 25 años & 97 & 29,5 \\
\hline Mujeres & 184 & 55,9 & Entre 26 a 33 años & 89 & 27,1 \\
\hline & & & Entre 34 a 41 años & 88 & 26,7 \\
\hline & & & Más de 41 años & 55 & 16,7 \\
\hline Antigüedad & & & Escolaridad & & \\
\hline Menos de 1 año & 90 & 27,4 & Sin estudios & 1 & 3,0 \\
\hline Entre 1 a 3 años & 107 & 32,5 & Primaria & 48 & 14,6 \\
\hline Entre 4 a 6 años & 70 & 21,3 & Secundaria & 179 & 54,4 \\
\hline Entre 7 a 10 años & 29 & 8,8 & Preparatoria & 88 & 26,7 \\
\hline Más de 10 años & 33 & 10,0 & Otro & 13 & 4,0 \\
\hline
\end{tabular}

Fuente: Elaboración propia. 


\subsection{Modelo de medida}

Cabe destacar que, de los sesenta ítems incluidos en el modelo de medición inicial, se eliminaron 6 por problemas de validez convergente, los cuales corresponden a capital psicológico (Cap10, Cap17, Cap18, Cap19, Cap20 y Cap22); así, quedaron en total 54 ítems en el modelo final. En la tabla 2, se exhiben los indicadores del modelo de medida con sus cargas, valores $t$, fiabilidad compuesta, alfa de Cronbach y análisis de varianza extraída (en adelante, AVE). Fornell y Larcker (1981) mencionan que, comúnmente, el punto de corte utilizado para las cargas es de 0,70, ya que indica que existe mayor varianza compartida entre el constructo y las variables latentes. En este modelo, los datos de las cargas en su mayoría muestran valores igual o superior a 0,70 , con la excepción de Just5 (0,617), Cap2 (0,696), Cap7 (0,663), Cap8 (0,679), Cap9 (0,671), Cap12 (0,663), Cap16 $(0,671)$, Sat6 $(0,629)$, Sat7 $(0,641)$ y Sat9 $(0,654)$; estos muestran valores inferiores a 0,70 . Sin embargo, Chin (1998) refiere que valores de 0,50 pueden ser aceptados siempre y cuando en el constructo se encuentren ítems con cargas altas. En todos los casos, los ítems son estadísticamente significativos, ya que sus valores t se encuentran entre 9,631 y 42,123, es decir, las cargas son significativas a un nivel de confianza del 99,9\%, pues los valores t muestran valores inferiores a 3,291.

La fiabilidad usualmente se mide con el alfa de Cronbach. Al respecto, Fornell y Bookstein (1982) recomiendan que los valores sean superiores a 0,70. Los valores alfa de Cronbach de las variables estudiadas cumplen con los siguientes criterios: justicia organizacional $(0,958)$, capital psicológico $(0,935)$ y satisfacción laboral $(0,962)$. Por su parte, Hair, Anderson, Tatham y Black (1999) afirman que existe otro indicador para medir la fiabilidad y que es una mejor medida de consistencia interna; dicho indicador se mide a través de la fiabilidad compuesta y el punto de corte recomendado es 0,70. En todos los casos, las variables cumplen con los siguientes criterios: justicia organizacional $(0,962)$, capital psicológico $(0,942)$ y satisfacción laboral $(0,965)$. Finalmente, se realizó el AVE. Dicho índice calcula la varianza que los constructos obtienen a partir de cada uno de sus indicadores. Chin (1998), y Gefen y Straub (2005) recomiendan que los valores sean superiores a 0,50 , lo cual revela que más del $50 \%$ de la varianza de cada uno de los constructos es explicada a partir de sus indicadores. Es así como justicia organizacional $(0,614)$, capital psicológico $(0,505)$ y satisfacción laboral $(0,559)$ cumplen con el criterio recomendado.

En la tabla 3, se presentan los indicadores de la validez discriminante a partir del AVE y HeterotraitMonotrait Ratio (en adelante, HTMT). En el primer caso, Fornell y Larcker (1981) dicen que existe validez discriminante entre el constructo y las variables latentes cuando la raíz cuadrada de la varianza extraída es mayor que la varianza compartida. En este caso, sobre la diagonal se presentan los indicadores AVE. Se aprecia que en todos los casos el indicador AVE es mayor que las correlaciones. Por ello, se afirma que existe validez discriminante. En el segundo caso, Henseler, Ringle y Sarstedt (2015) utilizan el indicador HTMT, que valora las correlaciones entre los indicadores que evalúan constructos distintos. Existen dos formas para evaluar la presencia de validez: a través de criterio o como prueba estadística. En este caso, se evaluó como criterio y se eligió como punto de corte el valor 0,85 . Se puede apreciar que los ratios cumplen con el indicador, por lo cual se afirma que existe validez discriminante. 
Tabla 2. Indicadores del modelo de medición

\begin{tabular}{|c|c|c|c|c|c|c|}
\hline \multicolumn{2}{|c|}{ Ítem } & Cargas & Valor t & $\begin{array}{l}\text { Alfa de } \\
\text { Cronbach }\end{array}$ & $\begin{array}{l}\text { Fiabilidad } \\
\text { compuesta }\end{array}$ & $\begin{array}{l}\text { Análisis } \\
\text { de AVE }\end{array}$ \\
\hline \multicolumn{4}{|c|}{ Justicia organizacional } & 0,958 & 0,962 & 0,614 \\
\hline \multicolumn{4}{|c|}{ En la empresa en la que laboro, las recompensas } & & & \\
\hline Just1 & en términos generales, en este trabajo, son bastante justas. & 0,745 & $24,476^{* * *}$ & & & \\
\hline Just2 & que recibo son de acuerdo a mi desempeńo. & 0,763 & $23,134^{* * *}$ & & & \\
\hline Just3 & muestran el esfuerzo que hago por mi trabajo. & 0,796 & $30,961^{* * *}$ & & & \\
\hline \multicolumn{4}{|c|}{ En la empresa } & & & \\
\hline Just4 & $\begin{array}{l}\text { puedo expresar mi punto de vista ante los procedimientos usados para } \\
\text { la recompensa }\end{array}$ & 0,746 & $24,604^{* * *}$ & & & \\
\hline Just5 & he intervenido en las recompensas a partir de los procedimientos. & 0,617 & $14,575^{* * *}$ & & & \\
\hline Just6 & las recompensas se aplican de manera permanente. & 0,797 & $35,142^{* * *}$ & & & \\
\hline Just7 & las recompensas se aplican de manera apropiada. & 0,816 & $42,123^{* * *}$ & & & \\
\hline Just8 & los procedimientos se basan en información correcta. & 0,814 & $34,450^{* * *}$ & & & \\
\hline \multicolumn{4}{|c|}{ Mi supervisor } & & & \\
\hline Just9 & me trata de manera educada. & 0,803 & $27,191^{* * *}$ & & & \\
\hline Just10 & me trata con dignidad. & 0,785 & $23,600^{* * *}$ & & & \\
\hline Just11 & me trata con respeto. & 0,768 & $22,133^{* * *}$ & & & \\
\hline Just12 & evita comentarios inapropiados. & 0,827 & $36,077^{* * *}$ & & & \\
\hline Just13 & es sincero cuando habla conmigo & 0,788 & $21,837^{* * *}$ & & & \\
\hline Just14 & $\begin{array}{l}\text { me explica detalladamente los procedimientos que se utilizan para } \\
\text { recompensar mi trabajo. }\end{array}$ & 0,826 & $35,301^{* * *}$ & & & \\
\hline Just 15 & me comunica de manera oportuna detalles relacionados con mi trabajo. & 0,780 & $23,875^{* * *}$ & & & \\
\hline Just16 & considera mis necesidades específicas para comunicarse conmigo. & 0,844 & $35,645^{* * *}$ & & & \\
\hline \multicolumn{4}{|c|}{ Capital psicológico } & 0,935 & 0,942 & 0,505 \\
\hline Cap1 & Me siento seguro analizando un problema para encontrar una solución. & 0,727 & $23,707^{* * *}$ & & & \\
\hline Cap2 & $\begin{array}{l}\text { Me siento seguro al representar a mi área de trabajo en reuniones con } \\
\text { los superiores. }\end{array}$ & 0,696 & $20,390^{* * *}$ & & & \\
\hline Cap3 & $\begin{array}{l}\text { Me siento seguro al contribuir a las decisiones sobre las estrategias de la } \\
\text { empresa. }\end{array}$ & 0,734 & $28,417^{* * *}$ & & & \\
\hline Cap4 & Me siento seguro al ayudar a establecer objetivos en mi área de trabajo. & 0,752 & $26,840^{* * *}$ & & & \\
\hline Cap5 & Me siento seguro al presentar información a mi grupo de trabajo & 0,793 & $37,637^{* * *}$ & & & \\
\hline Cap6 & $\begin{array}{l}\text { Si me encontrara presionado en el trabajo, podría pensar en muchas } \\
\text { maneras para salir de la situación. }\end{array}$ & 0,716 & $15,870^{* * *}$ & & & \\
\hline Cap7 & En la actualidad, persigo mis objetivos laborales. & 0,663 & $13,015^{* * *}$ & & & \\
\hline Cap8 & Hay muchas maneras de solucionar los problemas. & 0,679 & $14,276^{* * *}$ & & & \\
\hline Cap9 & Generalmente, sé cómo manejar los problemas en el trabajo. & 0,671 & $16,186^{* * *}$ & & & \\
\hline Cap11 & Por lo general, tomo con calma las cosas estresantes en el trabajo. & 0,712 & $15,985^{* * *}$ & & & \\
\hline
\end{tabular}




\begin{tabular}{|c|c|c|c|c|c|c|}
\hline \multicolumn{2}{|r|}{ Ítem } & \multirow{2}{*}{\begin{tabular}{|c|} 
Cargas \\
0,663 \\
\end{tabular}} & \multirow{2}{*}{\begin{tabular}{|c|} 
Valor t \\
$15,107^{* * *}$ \\
\end{tabular}} & $\begin{array}{l}\text { Alfa de } \\
\text { Cronbach }\end{array}$ & $\begin{array}{c}\text { Fiabilidad } \\
\text { compuesta }\end{array}$ & $\begin{array}{l}\text { Análisis } \\
\text { de AVE }\end{array}$ \\
\hline Cap12 & Siento que puedo manejar muchas cosas a la vez en este trabajo. & & & & & \\
\hline Cap13 & En este momento, me siento exitoso en el trabajo. & 0,731 & $21,823^{* * *}$ & & & \\
\hline Cap14 & Se me ocurren maneras diferentes de alcanzar los objetivos laborales. & 0,738 & $20,102^{* * *}$ & & & \\
\hline Cap15 & Siempre veo el lado positivo de las cosas con respecto a mi trabajo. & 0,710 & $15,036^{* * *}$ & & & \\
\hline Cap16 & Soy optimista con lo que me sucederá en mi trabajo en el futuro. & 0,671 & $13,010^{* * *}$ & & & \\
\hline Cap21 & $\begin{array}{l}\text { Cuando las cosas son inciertas para mí en el trabajo, generalmente, } \\
\text { espero lo mejor. }\end{array}$ & 0,705 & $15,403^{* * *}$ & & & \\
\hline \multicolumn{4}{|c|}{ Satisfacción laboral } & 0,962 & 0,965 & 0,559 \\
\hline \multicolumn{4}{|c|}{ Este trabajo } & & & \\
\hline Sat1 & me ofrece oportunidades para realizar las actividades en que destaco. & 0,758 & $25,311^{* * *}$ & & & \\
\hline Sat2 & me ofrece oportunidades para realizar las actividades que me gustan. & 0,751 & $24,913^{* * *}$ & & & \\
\hline Sat3 & me produce satisfacción. & 0,752 & $22,160^{* * *}$ & & & \\
\hline \multicolumn{4}{|c|}{ Estoy satisfecho con } & & & \\
\hline Sat4 & el salario que recibo. & 0,736 & $21,176^{* * *}$ & & & \\
\hline Sat5 & los objetivos, metas y tasas de producción que debo alcanzar. & 0,730 & $20,303^{* * *}$ & & & \\
\hline Sat6 & la limpieza, higiene y salubridad de mi lugar de trabajo & 0,629 & $11,774^{* * *}$ & & & \\
\hline Sat7 & el entorno físico y el espacio con el que cuento en mi lugar de trabajo. & 0,641 & $12,560^{* * *}$ & & & \\
\hline Sat8 & la iluminación de mi lugar de trabajo. & 0,573 & $9,631^{* * *}$ & & & \\
\hline Sat9 & la ventilación de mi lugar de trabajo. & 0,654 & $13,077^{* * *}$ & & & \\
\hline Sat10 & las oportunidades de formación que me ofrece la empresa. & 0,732 & $19,364^{* * *}$ & & & \\
\hline Sat11 & las oportunidades de promoción que tengo. & 0,798 & $29,784^{* * *}$ & & & \\
\hline Sat12 & las relaciones personales con mis supervisores. & 0,759 & $20,976^{* * *}$ & & & \\
\hline Sat13 & la supervisión que ejercen sobre mí. & 0,801 & $28,712^{* * *}$ & & & \\
\hline Sat14 & la frecuencia con que soy supervisado. & 0,800 & $30,259^{* * *}$ & & & \\
\hline Sat15 & la forma en que mis supervisores evalúan mi trabajo. & 0,803 & $33,490^{* * *}$ & & & \\
\hline Sat16 & la igualdad y justicia de trabajo que recibo de mi empresa. & 0,788 & $28,185^{* * *}$ & & & \\
\hline Sat17 & el apoyo que recibo de mis supervisores. & 0,796 & $31,289^{* * *}$ & & & \\
\hline Sat18 & $\begin{array}{l}\text { la capacidad para decidir yo mismos aspectos importantes de mi } \\
\text { trabajo. }\end{array}$ & 0,794 & $27,882^{* * *}$ & & & \\
\hline Sat19 & mi participación en las decisiones de mi área de trabajo. & 0,814 & $36,393^{* * *}$ & & & \\
\hline Sat20 & $\begin{array}{l}\text { mi participación en las decisiones importantes de mi grupo para la } \\
\text { empresa. }\end{array}$ & 0,766 & $24,075^{* * *}$ & & & \\
\hline Sat21 & el grado de mi empresa con las leyes laborales. & 0,760 & $23,506^{* * *}$ & & & \\
\hline Sat22 & $\begin{array}{l}\text { la forma en que se da la negociación en mi trabajo sobre aspectos } \\
\text { laborales. }\end{array}$ & 0,764 & $22,856^{* * *}$ & & & \\
\hline
\end{tabular}

*** Significativo con un alfa de 0,001 , si $t>3,291$, para una prueba de dos colas Fuente: Elaboración propia a partir de resultados de SmartPLS. 
Tabla 3. Validez discriminante

\begin{tabular}{|l|c|c|c|c|c|c|}
\hline \multirow{2}{*}{ Constructos } & \multicolumn{3}{|c|}{ Criterio AVE } & \multicolumn{2}{c|}{ Indicador HTMT } \\
\cline { 2 - 7 } & Capital psicológico & Justicia & Satisfacción & Capital psicológico & Justicia & Satisfacción \\
\hline Capital psicológico & 0,711 & & & & & \\
\hline Justicia & 0,671 & 0,784 & & 0,696 & \\
\hline Satisfaccion & 0,774 & 0,783 & 0,748 & 0,809 & 0,809 & \\
\hline
\end{tabular}

Fuente: Elaboración propia.

Figura 1. Modelo estructural

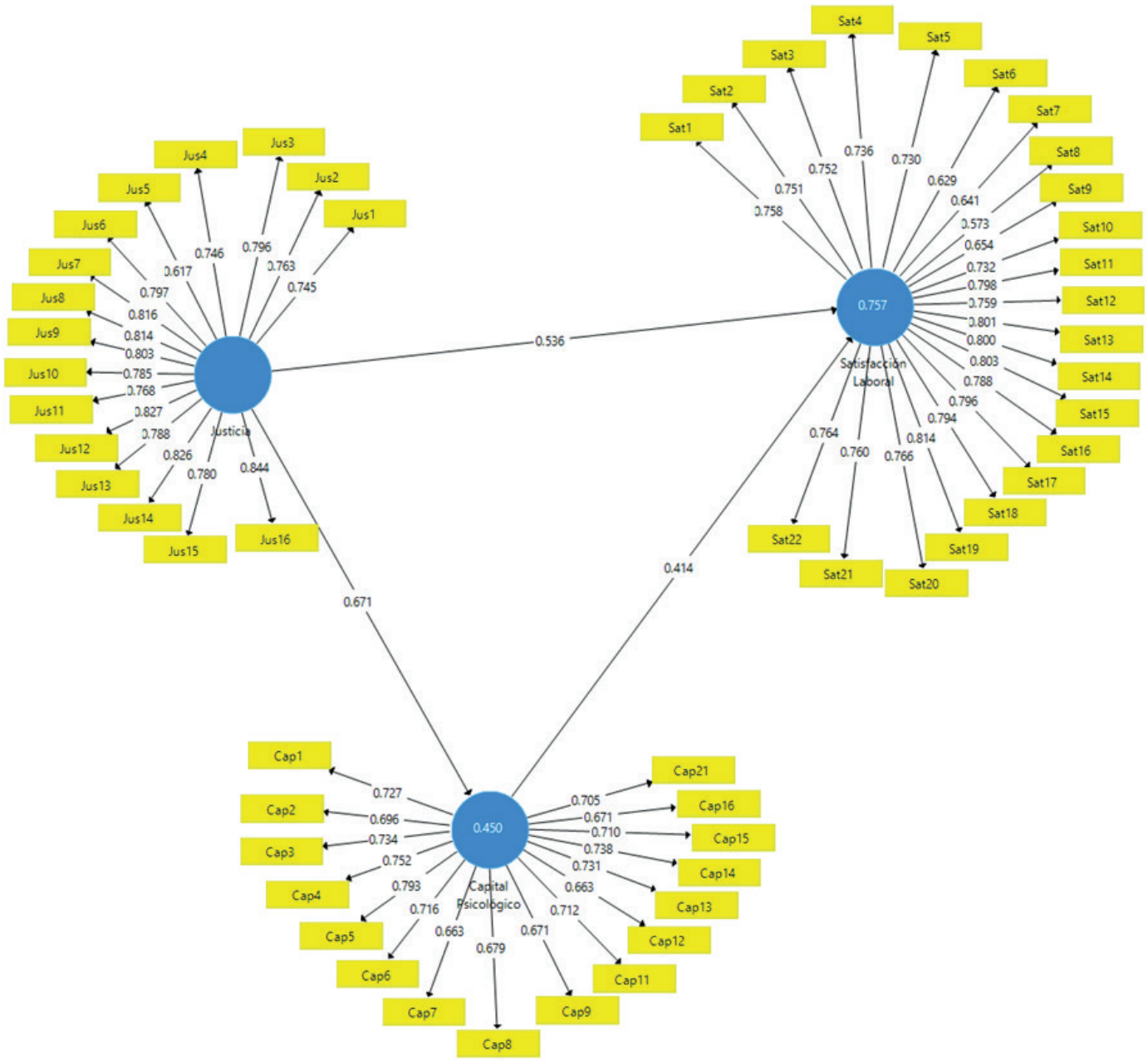

*** Significativo con un alfa de 0,001 , si $\mathrm{t}>3,291$, para una prueba de dos colas Fuente: Elaboración propia a partir de SmartPLS 3. 
En la figura 1, se exhibe el modelo estructural en el que se observan los coeficientes paths y los valores de $\mathrm{r}^{2}$. Se aprecia que los paths planteados cumplen con el criterio aceptable de 0,20, ademas de ser significativos estadisticamente: justicia-satisfacción laboral $(0,536 ; t=10,588)$, justicia-capital psicológico $(0,671$; $\mathrm{t}=17,565)$, capital psicológico-satisfacción laboral $(0,414 ; t=8,718)$. Por su parte, Chin (1998) señala que el coeficiente $r^{2}$ indica la medida en la que la variable endógena es explicada por las variables predictoras. En este caso de forma moderada, la justicia explica el capital psicológico $\left(\mathrm{r}^{2}=0,450\right)$ y, sustancialmente, la satisfacción laboral se explica a través del capital psicológico $\left(r^{2}=0,757\right)$.

Como se comentó al inicio, la investigación partió del planteamiento de tres hipótesis: 1) H1: la justicia incide positiva y significativamente sobre la satisfacción laboral, 2) H2: la justicia incide positiva y significativamente sobre el capital psicológico y 3) H3: el capital psicológico influye significativamente sobre la satisfacción laboral. En la tabla 4, se aprecia que ninguna de ellas se rechazó, ya que se evidencia que la justicia incide positiva y significativamente sobre la satisfacción laboral $\left(\mathrm{H} 1 ; \mathrm{p}=0,000^{* * *}\right)$ y el capital psicológico $\left(\mathrm{H} 2 ; \mathrm{p}=0,000^{* * *}\right)$; además, el capital psico- lógico influye significativamente sobre la satisfacción laboral $\left(\mathrm{H} 3 ; \mathrm{p}=0,000^{* * *}\right)$. En los tres casos, se aprecian valores $\mathrm{P}<0,05$ y valores $t$ superiores a 3,291 , por lo que se afirma que los tres planteamientos son fiables a un nivel de confianza del 99,9\%.

\section{Conclusiones}

Este trabajo de investigación analizó el contexto de la industria manufacturera, con el objetivo de indagar si la justicia organizacional impacta sobre el capital psicológico y la satisfacción laboral de los empleados de nivel operativo que laboran en una empresa del ramo automotriz. Los resultados evidencian que las relaciones planteadas inciden significativamente: la justicia incide tanto directa como indirectamente a través de la mediación del capital psicológico en la satisfacción laboral de los empleados que laboran en el entorno estudiado.

En el contexto de la industria automotriz, la justicia impacta significativamente en la satisfacción de los empleados de nivel operativo; es decir, cuando los empleados perciben que el trabajo asignado es equitativo, son considerados en los procesos de recompensas y estas son llevadas a cabo, y se sentirán satisfechos en el entorno laboral. Esto se explica porque los sujetos

Tabla 4. Prueba de hipótesis

\begin{tabular}{|c|c|c|c|c|}
\hline Hipótesis & Coeficiente path & Valor t & Valor $\mathrm{p}$ & Resultado \\
\hline $\begin{array}{l}\text { H1: La justicia incide positiva y significativamente sobre la } \\
\text { satisfacción laboral. }\end{array}$ & 0,536 & $10,588^{* * *}$ & $0,000^{* * *}$ & No rechazo \\
\hline $\begin{array}{l}\text { H2: La justicia incide positiva y significativamente sobre el } \\
\text { capital psicológico. }\end{array}$ & 0,671 & $17,565^{* * *}$ & $0,000^{* * *}$ & No rechazo \\
\hline $\begin{array}{l}\text { H3: El capital psicológico influye significativamente sobre la } \\
\text { satisfacción laboral. }\end{array}$ & 0,414 & $8,718^{* * *}$ & $0,000^{* * *}$ & No rechazo \\
\hline
\end{tabular}

*** Significativo con un nivel de significancia del 99,9\%, si $\mathrm{P}<0,05$

*** Significativo con un alfa de 0,001, si t $>3,291$, para una prueba de dos colas Fuente: Elaboración propia a partir de resultados de SmartPLS 3. 
de estudio son tomados en cuenta en las decisiones que les concierne. Además, cuando prevalece un trato justo y se informan oportunamente las decisiones que atañen a los trabajadores, su satisfacción se verá fortalecida. Esto se verá reflejado en los resultados de la organización, particularmente, en los resultados operacionales. Los hallazgos encontrados coinciden con lo planteado por Karam et al. (2019), Lambert et al. (2020), Pérez et al. (2014), y Wright y Bonett (2007). Estos autores manifiestan que un entorno de justicia propicia un ambiente agradable y de satisfacción.

Este estudio también muestra que la justicia incide en el capital psicológico y que este tiene un efecto positivo en el trabajo que realizan los operadores. Se considera que el capital psicológico aumenta y se fortalece si los empleados perciben que son tratados con respeto y equidad, así como cuando las recompensas son consideradas justas, es decir, que el trabajo se vea compensado con el pago percibido. Así, los líderes reconocen el esfuerzo que los trabajadores realizan. Cuando esto sucede, los sujetos proponen buenas ideas sobre cómo realizar el trabajo, y se involucran en establecer, realizar y lograr los objetivos. Además, los empleados pueden contribuir en las decisiones sobre las estrategias de la empresa y, por ende, ser exitosos en las tareas que realizan. Los resultados de la investigación coinciden con lo planteado por Eom et al. (2016), y Singh y Singh (2019), que señalan que los empleados que perciben justicia revelan comportamientos positivos directamente beneficiosos para la organización.

Es importante destacar que tanto la justicia como el capital psicológico provocan en los empleados satisfacción en el lugar de trabajo, dado que, al percibir que prevalece la justicia, se manifiestan emociones y comportamientos positivos que impactan directamente en la satisfacción de las personas. Esto se ve corroborado por lo señalado por Perugini y Solano (2019),
Saraswati (2019), y Singh y Singh (2019). Por otro lado, cabe considerar que, cuando los sujetos perciben injusticia en el entorno laboral, exteriorizan insatisfacción (Heidari \& Saeedi, 2012; Saraswati, 2019).

Esta investigación tiene limitaciones, dado que se utilizó un diseño transversal y una población en específico. Por lo tanto, no se pueden generalizar los resultados. Se considera conveniente contemplar otros sujetos de estudio, como personal indirecto y de confianza, así como mayores rangos de antigüedad en el lugar de trabajo y otro tipo de industria. Además, es importante realizar estudios longitudinales.

\section{Referencias bibliográficas}

Alvarez, L., de Miguel, M., Noda, E., Alvarez, L. F., \& Galcerán, G. (2016). Diagnóstico de la satisfacción laboral en una entidad asistencial hospitalaria. Revista Cubana de Salud Pública, 42, 407-417.

Azanza, G., Domínguez, Á. J., Moriano, J. A., \& Molero, F. J. (2014). Capital psicológico positivo. Validación del cuestionario PCQ en España. Anales de PsicologialAnnals of Psychology, 30(1), 294-301. https://doi. org/10.6018/analesps.30.1.153631

Bautista, S. J., \& Peralta, C. M. R. (2017). La inclusión de las pymes en la cadena de valor de la industria automotriz en México en el marco del Tratado Trans-Pacífico (TTP). Economía Informa, 403, 46-65. https:// doi.org/10.1016/j.ecin.2017.05.004

Bogler, R., \& Somech, A. (2019). Psychological Capital, Team Resources and Organizational Citizenship Behavior. The Journal of Psychology, 153(8), 784-802. https://doi.org/10.1080/00223980.2019.1614515

Bouazzaoui, M., Wu, H.-J., Roehrich, J. K., Squire, B., \& Roath, A. S. (2020). Justice In Inter-Organizational Relationships: A Literature Review and Future Research Agenda. Industrial Marketing Management, 87, 128-137. https://doi.org/10.1016/j.indmarman.2020.02.003

Contabilidad y Negocios (16) 31, 2021 / e-ISSN 2221-724X 
Bravo, M. J., Peiró, J., \& Rodríguez, I. (1996). Satisfacción laboral. Tratado de Psicología del Trabajo, 1, 343-394.

Chin, W. W. (1998). Issues and Opinion on Structural Equation Modeling. MIS Quarterly, 22(1), 7-16.

Colquitt, J. A. (2001). On the Dimensionality of Organizational Justice: A Construct Validation of a Measure. Journal of Applied Psychology, 86(3), 386-400. https:// doi.org/10.1037/0021-9010.86.3.386

Corcione, A. C. (2015). Capital psicológico: un constructo fundacional dentro de la psicología organizacional positiva. Revista Cientifica de Ciencias de la Salud, 8(2), 50-55. https://doi.org/10.17162/rccs.v8i2.473

Cropanzano, R., Bowen, D. E., \& Gilliland, S. W. (2007). The Management of Organizational Justice. Academy of Management Perspectives, 21(4), 34-48. https://doi. org/10.5465/amp.2007.27895338

De Boer, E. M., Bakker, A. B., Syroit, J. E., \& Schaufeli, W. B. (2002). Unfairness at Work as a Predictor of Absenteeism. Journal of Organizational Behavior: The International Journal of Industrial, Occupational and Organizational Psychology and Behavior, 23(2), 181197. https://doi.org/10.1002/job.135

Dipp, A. J., Flores, J. A. T., \& Gutiérrez, R. V. (2010). Satisfacción laboral y compromiso institucional de los docentes de posgrado. Diálogos Educativos, (19), 119-130.

Eom, J., Hwang, C., \& Song, Y. (2016). The Effect of the Corporate Employee's Perception of Organizational Justice on Psychological Capital, Occupational Satisfaction, and Organizational Commitment: Mainly on the Workers in Construction and Manufacturing. Journal of the Korea Society of Digital Industry and Information Management, 12(3), 273-297. https:// doi.org/10.17662/ksdim.2016.12.3.273

Escajadillo, S. H. (2013). Clima organizacional y satisfacción laboral en la empresa fyd inversiones SAC. Industrial Data, 16(2), 75-78. https://doi.org/10.15381/ idata.v16i2.11924
Ewen, R. B., Smith, P. C., \& Hulin, C. L. (1966). An Empirical Test of the Herzberg Two-Factor Theory. Journal of Applied Psychology, 50(6), 544-550. https:// doi.org/10.1037/h0024042

Fornell, C., \& Bookstein, F. L. (1982). Two Structural Equation Models: LISREL and PLS Applied to Consumer Exit-Voice Theory. Journal of Marketing Research, 19(4), 440-452. https://doi.org/10.1177/ 002224378201900406

Fornell, C., \& Larcker, D. F. (1981). Evaluating Structural Equation Models with Unobservable Variables and Measurement Error. Journal of Marketing Research, 18(1), 39-50.https://doi.org/10.1177/002224378101800104

Gabriunas, I. L. P. (2010). La influencia del desempeńo social corporativo en la satisfacción laboral de los empleados: una revisión teórica desde una perspectiva multinivel. Estudios Gerenciales, 26(116), 63-81. https://doi.org/10.1016/S0123-5923(10)70123-6

Gefen, D., \& Straub, D. (2005). A Practical Guide to Factorial Validity Using PLS-Graph: Tutorial and Annotated Example. Communications of the Association for Information Systems, 16, 91-109. https://doi. org/10.17705/1CAIS.01605

Greenberg, J. (1990). Organizational Justice: Yesterday, Today, and Tomorrow. Journal of Management, 16(2), 399432. https://doi.org/10.1177/014920639001600208

Hair, J. F., Anderson, R. F., Tatham, R. L., \& Black, W. C. (1999). Análisis Multivariante (5a ed.). Madrid: Prentice Hall Iberia.

Heidari, S. A., \& Saeedi, N. (2012). Studying the Role of Organizational Justice on Job Satisfaction (Case study: An Iranian company). Journal of Basic and Applied Scientific Research, 2(7), 6459-6465.

Henseler, J., Ringle, C. M., \& Sarstedt, M. (2015). A New Criterion for Assessing Discriminant Validity in Variance-Based Structural Equation Modeling. Journal of the Academy of Marketing Science, 43(1), 115-135. https://doi.org/10.1007/s11747-014-0403-8 
Index Juárez. (2020). Información estadística mensual: 3 de julio 2020. Recuperado de https://indexjuarez.com/ wp-content/uploads/2020/07/Julio-3.pdf [Consulta: 30 de noviembre de 2020].

Jiménez, A. E., Jara, M. J., \& Celis, E. R. M. (2012). Burnout, apoyo social y satisfacción laboral en docentes. Psicologia Escolar y Educacional, 16(1), 125-134. https://doi. org/10.1590/S1413-85572012000100013

Karam, E. P., Hu, J., Davison, R. B., Juravich, M., Nahrgang, J. D., Humphrey, S. E., \& Scott DeRue, D. (2019). Illuminating the 'Face' of Justice: A MetaAnalytic Examination of Leadership and Organizational Justice. Journal of Management Studies, 56(1), 134-171. https://doi.org/10.1111/joms.12402

Lambert, E. G., Keena, L. D., Leone, M., May, D., \& Haynes, S. H. (2020). The Effects of Distributive and Procedural Justice on Job Satisfaction and Organizational Commitment Ofcorrectional Staff. The Social Science Journal, 57(2) 1-12. https://doi.org/10.1016/j. soscij.2019.02.002

López, S. M., del Río, E. F., \& Villagrasa, P. J. R. (2018). La satisfacción de los empleados públicos con sus supervisores: El papel de la transparencia y la justicia organizacional. Acciones e Investigaciones Sociales, (38), 81-97. https://doi.org/10.26754/ojs_ais/ ais. 2018382977

Luthans, F., Avolio, B. J., Avey, J. B., \& Norman, S. M. (2007). Positive Psychological Capital: Measurement and Relationship with Performance and Satisfaction. Personnel Psychology, 60(3), 541-572. https://doi. org/10.1111/j.1744-6570.2007.00083.x

Luthans, F., Luthans, K. W., \& Luthans, B. C. (2004). Positive Psychological Capital: Beyond Human and Social Capital. Business Horizons, 47(1), 45-50. https://doi. org/10.1016/j.bushor.2003.11.007

Luthans, F., Youssef, C. M., \& Avolio, B. J. (2007). Psychological Capital: Developing the Human Competitive Edge. Oxford: Oxford University Press.
Martín, P. A. (2006). Diferencias en la percepción de la satisfacción laboral en una muestra de personal de administración. Boletín de Psicología, 88, 49-63.

Meliá, J., \& Peiró, J. (1989). La medida de la satisfacción laboral en contextos organizacionales: El Cuestionario de Satisfacción S20/23. Psicologemas, 5(2), 59-74.

Mladinic, A., \& Isla, P. (2002). Justicia Organizacional: Entendiendo la Equidad en las Organizaciones Organizational Justice: Understanding Equity in Organizations. Psykhe, 11(2), 171-179.

Mladinic, A., \& Isla, P. (2011). Justicia organizacional: entendiendo la equidad en las organizaciones. Psykhe, 11(2).

Montalbán, R. L. R., Lugo, M. M., \& Soria, M. S. (2014). Justicia organizacional, engagement en el trabajo y comportamientos de ciudadanía organizacional: una combinación ganadora. Universitas Psychologica, 13(3). https://doi.org/10.11144/Javeriana.UPSY13-3.joet

Newstrom, J. (2011). Comportamiento humano en el trabajo. México, D.F.: McGraw-Hill: Interamericana.

Omar, A., Salessi, S., \& Urteaga, F. (2014). Diseńo y validación de la escala CapPsi para medir capital psicológico. Liberabit, 20(2), 315-323.

Paek, S., Schuckert, M., Kim, T. T., \& Lee, G. (2015). Why is Hospitality Employees' Psychological Capital Important? The Effects of Psychological Capital on Work Engagement and Employee Morale. International Journal of Hospitality Management, 50, 9-26. https://doi.org/10.1016/j.ijhm.2015.07.001

Peńa, M. C., Olloqui, A. M., \& Aguilar, A. (2013). Relación de factores en la satisfacción laboral de los trabajadores de una pequeña empresa de la industria metal-mecánica. Revista Internacional Administracion \& Finanzas, 6(3), 115-128.

Pérez, J. P., Herrera, R. F., Torres, E. M., \& Hernández, R. H. (2014). Validez y confiabilidad de la escala de justicia organizational de Niehoff y Moorman en población

Contabilidad y Negocios (16) 31, 2021 / e-ISSN 2221-724X 
mexicana. Contaduría y Administración, 59(2), 97-120. https://doi.org/10.1016/S0186-1042(14)71256-2

Perugini, M. L. L., \& Solano, A. C. (2019). Virtudes organizacionales y capital psicológico como predictores positivos de satisfacción y performance laboral. Revista de Psicología, 15(29), 22-35.

Robbins, S. (2004). Comportamiento organizacional (10 ed.). México, D F.: Pearson Educación.

Robbins, S., \& Judge, T. (2009). Comportamiento organizacional (13 a ed.). Naucalpan de Juárez:. Pearson Educación.

Salazar, J. G. N., Cosiun, G. J. B., \& Torres, D. R. (2019). Relación del clima organizacional con la satisfacción laboral en una empresa del sector de la construcción. Revista Cientifica ECOCIENCIA, 6(1), 1-24. https:// doi.org/10.21855/ecociencia.61.184

Salessi, S. M., \& Omar, A. G. (2017). Capital psicológico y regulación emocional en el trabajo: el rol mediador de la satisfacción laboral. Estudos de Psicologia, 22(1), 89-98. https://doi.org/10.22491/1678-4669.20170010

Saraswati, K. D. (2019). Work Engagement: The Impact of Psychological Capital and Organizational Justice and its Influence on Turnover Intention. Journal of Management and Marketing Review, 4(1), 86-91. https:// doi.org/10.35609/jmmr.2019.4.1(12)
Shibaoka, M., Takada, M., Watanabe, M., Kojima, R., Kakinuma, M., Tanaka, K., \& Kawakami, N. (2010). Development and Validity of the Japanese Version of the Organizational Justice Scale. Industrial Health, 4(1), 66-73. https://doi.org/10.2486/indhealth.48.66

Singh, P., \& Loncar, N. (2010). Pay Satisfaction, Job Satisfaction and Turnover Intent. Relations Industrielles/ Industrial Relations, 65(3), 470-490. https:/doi. org/10.7202/044892ar

Singh, S. K., \& Singh, A. P. (2019). Interplay of Organizational Justice, Psychological Empowerment, Organizational Citizenship Behavior, and Job Satisfaction in the Context of Circular Economy. Management Decision. https://doi.org/10.1108/MD-09-2018-0966

Wright, T. A., \& Bonett, D. G. (2007). Job Satisfaction and Psychological Well-Being as Nonadditive Predictors of Workplace Turnover. Journal of Management, 33(2), 141-160.https://doi.org/10.1177/0149206306297582

Fecha de recepción: 04 de agosto de 2020 Fecha de aceptación: 07 de abril de 2021 Correspondencia: al143346@alumnos.uacj.mx al137258@alumnos.uacj.mx maría.vargas@uacj.mx karla.gomez@uacj.mx 